\title{
Mass Hierarchy Determination Using Neutrinos from Multiple Reactors
}

\author{
Emilio Ciuffoli ${ }^{1 *}$, Jarah Evslin $^{1 \dagger}$ and Xinmin Zhang ${ }^{2,1 \ddagger}$ \\ 1) TPCSF, IHEP, Chinese Acad. of Sciences \\ 2) Theoretical physics division, IHEP, Chinese Acad. of Sciences \\ YuQuan Lu 19(B), Beijing 100049, China
}

\begin{abstract}
We report the results of Monte Carlo simulations of a medium baseline reactor neutrino experiment. The difference in baselines resulting from the $1 \mathrm{~km}$ separations of Daya Bay and Ling Ao reactors reduces the amplitudes of 1-3 oscillations at low energies, decreasing the sensitivity to the neutrino mass hierarchy. A perpendicular detector location eliminates this effect. We simulate experiments under several mountains perpendicular to the Daya Bay/Ling Ao reactors, considering in particular the background from the TaiShan and YangJiang reactor complexes. In general the hierarchy can be determined most reliably underneath the 1000 meter mountain BaiYunZhang, which is $44.5 \mathrm{~km}$ from Daya Bay. If some planned reactors are not built then nearby 700 meter mountains at $47-51 \mathrm{~km}$ baselines gain a small advantage. Neglecting their low overhead burdens, hills near BaiMianShi or DongKeng would be the optimal locations. We use a weighted Fourier transform to avoid a spurious dependence on the high energy neutrino spectrum and find that a neural network can extract quantities which determine the hierarchy marginally better than the traditional $R L+P V$.
\end{abstract}

October 10, 2018

\footnotetext{
*ciuffoli@ihep.ac.cn

$\dagger$ jarah@ihep.ac.cn

${ }^{\ddagger}$ xmzhang@ihep.ac.cn
} 


\section{Introduction}

This year the Daya Bay [1,2] and RENO [3] experiments accurately determined $\theta_{13}$. While there had been hints $[4,5]$ that $\theta_{13}$ might be large in 2011 , in previous years the tight constraints placed by CHOOZ [6] and Palo Verde [7] meant that analyses of 1-3 oscillations often assumed a value of the mixing parameter $\sin ^{2}\left(2 \theta_{13}\right)$ an order of magnitude or more below the true value. The new, large value of $\theta_{13}$ has led to a reexamination of the longstanding belief that, with the known low value of $\Delta M_{21}^{2}$, it is not practical to measure the neutrino hierarchy at a medium baseline reactor experiment [8, 9]. Now not only is such a measurement in principle possible, but indeed it will be attempted within the next decade $[10,11,12]$.

Using the old value of $\theta_{13}$, the authors of Ref. [13] proposed an optimal baseline and analysis for determining the hierarchy, building upon the Fourier transform strategy of Ref. [14]. Then in Ref. [15] they performed a series of simulations to test these ideas. In Ref. [16] we have updated the analysis of Ref. [13] to the new value of $\theta_{13}$, including several important new effects such as the interference caused by the spacing between reactors in a reactor complex. In the current note we will update the simulations of Ref. [15] to the new value of $\theta_{13}$. We will apply the interference effect and also the spurious dependence of the Fourier transform on high energy neutrinos $[17,18]$ to study the optimal baseline for such an experiment as well as the optimal mountain under which such an experiment might be built in China's Guangdong province.

As the Daya Bay and Ling Ao reactor complexes enjoy 8 neutrino detectors, they are optimal neutrino sources for a medium baseline reactor experiment. However the distance between the Daya Bay reactor complex and the two Ling Ao complexes is more than a kilometer, which means that low energy reactor neutrinos can oscillate half of a phase while traveling from one to the other. As a result, if a detector is roughly along the line which separates these detectors, then the low energy 1-3 neutrino oscillation amplitude will decrease appreciably and so be difficult to observe. We will refer to this effect as interference, although the neutrinos from Daya Bay and Ling Ao are never coherent, the effect is caused by the addition of probabilities and not wavefunctions. Since the determination of the hierarchy at a medium baseline experiment [8] relies upon the observation of 1-3 oscillation peaks, and in particular the low energy peaks are necessary to break a degeneracy present in the high energy peaks $[9,17,16]$, we conclude that the interference effect hinders a determination of the hierarchy at the Daya Bay II reactor experiment locations proposed in Refs. [10, 12, 19]. 
On the other hand, these locations benefit from the flux of an additional reactor complex which may be approved and eventually built at HuiDong. As we will see below, the additional flux would suffice to make these the ideal sites (along with a site near DongKeng located between the TaiShan and YangJiang reactor complexes) except for the fact that we know of no mountain at a medium baseline which is equidistant from Daya Bay and HuiDong (TaiShan and YangJiang in the case of the DongKeng site). The maximum elevation at an equidistant site is only about 200-300 meters in both cases, meaning that backgrounds caused by atmospheric muons may significantly reduce the detector efficiency. Both are about $3 \mathrm{~km}$ from 500 meter mountains, but we will see that this $3 \mathrm{~km}$ baseline difference leads to another interference effect which makes experiments under these mountains essentially insensitive to the hierarchy, unless the direction in which a neutrino traveled can be sufficiently well established to determine from which reactor it originated with sufficiently high probability, for example if at least in some energy range one can improve upon the technique used by the CHOOZ collaboration in Ref. [20].

We will therefore find that the best locations are perpendicular to the Daya Bay and Ling Ao reactors, where this interference effect is absent. As there will be backgrounds from other reactors, for example, the TaiShan and YangJiang reactors which will begin to come into service next year, a short baseline is essential to optimize the signal to noise ratio. The more reactors that will be built, the shorter the optimal baseline will be. We have simulated various combinations of reactors and in general we find that the best location is the 1000 meter mountain BaiYunZhang which is $44.5 \mathrm{~km}$ from Daya Bay. However a number of 700 meter mountains are located within 10 kilometers of BaiYunZhang. These mountains offer somewhat larger baselines, which may be optimal if many of the planned reactors are not approved.

We begin in Sec. 2 with a description of our Monte Carlo and the way that we convert the observed neutrino spectrum into a determination of the neutrino mass hierarchy. We define a number of quantities which reflect features of the Fourier transform of the spectrum, generalizing those of Ref. [13], and then the hierarchy is determined by a linear combination of these quantities with weights that are optimized by a neural network. It was noted in Ref. [17] that the features of a Fourier transform are very sensitive to the reactor spectrum and to $\left|\Delta M_{32}^{2}\right|$. It was shown in Ref. [18] that this dependence yields no information about the hierarchy but can be eliminated using a weighted Fourier transform. We let the first layer of the neural network optimize these weights. In Sec. 4 we systematically study reactors at different baselines from single detectors, finding the optimal baseline to be in the 48-52 $\mathrm{km}$ range in this case. We also study the interference effect at various angles. Finally in Sec. 5 we describe the detector and reactor locations considered and present the results of 
our simulations. We find that even reactors $200 \mathrm{~km}$ away from the detector can appreciably impede the determination of the hierarchy so that, as a high signal to background ratio becomes essential, they reduce the optimal baseline from that found in Sec. 4. For simplicity and an easy comparison with earlier results, we do not use the neural network in the analysis in Sec. 5 but instead consider the analysis method of Ref. [13], although an application of our neural network to BaiYunZhang is described in Subsec. 3.4.

After this paper was written we received the preprint [21] which presents a $\chi^{2}$ analysis of the spectrum of a single reactor at a baseline of $60 \mathrm{~km}$. That study differs from ours in that it does not include statistical errors, thus as may be expected it found that about an order of magnitude less flux is required for a determination of the hierarchy with comparable confidence. Our results for single reactor experiments are, on the other hand, broadly compatible with the Fourier transform based analysis of Ref. [10] and with the $\chi^{2}$ analysis of Ref. [17].

\section{The Monte Carlo}

\subsection{The Parameters}

This paper builds upon the simulations of medium baseline neutrino oscillation in Refs. [15, 10]. For ease of comparing with the results of [10], we have adopted the same values for the neutrino mass matrix parameters

$$
\Delta M_{21}^{2}=7.59 \times 10^{-5} \mathrm{eV}^{2}, \quad\left|\Delta \mathrm{M}_{32}^{2}\right|=2.43 \times 10^{-3} \mathrm{eV}^{2}, \quad \sin ^{2}\left(2 \theta_{12}\right)=0.861
$$

In particular we have used Daya Bay's March result [1]

$$
\sin ^{2}\left(2 \theta_{13}\right)=0.092
$$

and not their updated result [2]. Similarly we have assumed an absolute detector energy resolution

$$
\sigma_{E}=.03 \sqrt{E_{p r}(\mathrm{MeV})}
$$

where the prompt energy $E_{p r}$ is related to the neutrino energy $E$ and the positron energy $E_{e}$ by

$$
E_{p r}=E_{e}+m_{e}=E-m_{n}+m_{p}+m_{e} \sim E-0.8 \mathrm{MeV}
$$




\subsection{The Simulation}

The overall reactor flux normalization is fixed to be that of Ref. [10]. It is asserted that the Daya Bay plus Ling Ao I and II reactor complexes, which together have 17.4 GW of thermal capacity, lead to the observation of 25,000 neutrinos at a 20 kton detector with a baseline of $58 \mathrm{~km}$. To be precise this does not fix the reactor flux normalization, but rather the overall normalization of the product of the reactor flux, the antineutrino cross section of the target, the detector efficiency and the effect of neutrino oscillations. This condition, together with the tree level inverse $\beta$ decay cross section in Ref. [23]

$$
\sigma(E)=0.0952 \times 10^{-42} \mathrm{~cm}^{2}\left(E_{e} \sqrt{E_{e}^{2}-m_{e}^{2}} / \mathrm{MeV}^{2}\right)
$$

and the oscillation probability $P_{e e}$

$$
\begin{aligned}
& P_{e e}=\sin ^{4}\left(\theta_{13}\right)+\cos ^{4}\left(\theta_{12}\right) \cos ^{4}\left(\theta_{13}\right)+\sin ^{4}\left(\theta_{12}\right) \cos ^{4}\left(\theta_{13}\right)+\frac{1}{2}\left(P_{12}+P_{13}+P_{23}\right) \\
& P_{12}=\sin ^{2}\left(2 \theta_{12}\right) \cos ^{4}\left(\theta_{13}\right) \cos \left(\frac{\Delta M_{21}^{2} L}{2 E}\right), \quad P_{13}=\cos ^{2}\left(\theta_{12}\right) \sin ^{2}\left(2 \theta_{13}\right) \cos \left(\frac{\left|\Delta M_{31}^{2}\right| L}{2 E}\right) \\
& P_{23}=\sin ^{2}\left(\theta_{12}\right) \sin ^{2}\left(2 \theta_{13}\right) \cos \left(\frac{\left|\Delta M_{32}^{2}\right| L}{2 E}\right)
\end{aligned}
$$

are then used to normalize the effective reactor flux $\Phi(E)$ per time per GW of thermal capacity. This reactor flux is effective in the sense that it is already multiplied by the efficiency of the detector.

With this optimistic normalization in hand, the average number density of antineutrinos at energy $E$ from a given reactor which would be observed during 3 years at a 20 kton detector at a baseline $L$ is then

$$
N_{\mathrm{th}}(E, L)=\frac{\Phi(E) \sigma(E) P_{e e}(L / E)}{4 \pi L^{2}} .
$$

In each 60 kton year experiment we simulate

$$
N_{\mathrm{th}}(L)=\int d E N_{\mathrm{th}}(E, L)
$$

neutrinos from each reactor. Thus our simulations differ from a real experiment in that the total neutrino flux normalization is fixed and is known precisely. We will recreate 120 year experiments by simply summing the neutrinos from pairs of 60 kton year experiments. To minimize the relative statistical errors between experiments with different exposure times, 120 kton year experiments will be created from 60 kton experiments reported on the same tables. 
As can be seen in Table 2, unlike Refs. $[15,10]$ our total neutrino flux in each experiment depends on $L$. In fact it increases faster than $1 / L^{2}$ as $L$ is decreased from $58 \mathrm{~km}$ because there is less loss due to 1-2 neutrino oscillations. This means that at short baselines, such as $40-50 \mathrm{~km}$, our simulations allow a precise determination of the high energy peaks, which are hardly depleted by 1-2 oscillation. The energies $2 \pi / \Delta M_{\mathrm{eff}}^{2}$ of these peaks determine the effective mass splitting $[24,16]$

$$
\Delta M_{\mathrm{eff}}^{2}=\cos ^{2}\left(\theta_{12}\right)\left|\Delta M_{31}^{2}\right|+\sin ^{2}\left(\theta_{12}\right)\left|\Delta M_{32}^{2}\right| .
$$

As a result we will see that our simulations favor shorter baselines than those preferred in Refs $[15,10]$. We will also see that shorter baselines are preferred as they reduce the fractional backgrounds from distant reactors, backgrounds not included in previous studies.

Once we have fixed the numbers and energies of the neutrinos arriving at our detector, the finite resolution $\sigma_{E}$ of the detector is applied. With infinite luminosity the observed neutrino spectrum $P_{\text {obs }}(E)$ would be the theoretical spectrum convoluted with a Gaussian of width $\sigma_{E}$. To implement this effect at a finite luminosity we shift the energy of a neutrino by a random variable $\delta$ with Gaussian probability density $\exp \left(-\delta^{2} / 2 \sigma_{\mathrm{E}}^{2}\right) / \sqrt{2 \pi \sigma_{\mathrm{E}}}$.

Thus our simulation correctly accounts for statistical errors in the determination of the energy due to the finite number of photoelectrons detected. However it does not take into account a systematic nonlinear error in the determination of energy. While linear errors are harmless to the Fourier analysis that will be performed later, they simply shift the points, even a small nonlinear uncertainty can alter [22] or destroy [17] the effectiveness of such an analysis. In practice the energy can be calibrated, at least at some energies and at some points inside of the detector, by inserting radioactive sources. A peak by peak analysis of the spectrum can be performed just using peaks at these energies [16] but this requires more flux than a Fourier analysis.

\subsection{Improvements over Previous Simulations}

Although the simulation does not account for the unknown nonlinear response of the detector, it does include an interference effect [16] which has so far not been simulated in the literature. The reactor complex at Daya Bay is separated by more than a kilometer from the reactor complexes Ling Ao I and Ling Ao II. While this set of reactors is in general an ideal choice for medium baseline experiments, given the high flux and the existence of 8 short baseline detectors, in general the 1-3 oscillations from Ling Ao and Daya Bay will be out of phase at low energies. The measurement of 1-3 oscillations at these low energies are essential to break a degeneracy which prohibits the determination of the neutrino mass hierarchy $[9,17,16]$. Thus 
such interference poses a severe problem for the determination of the hierarchy. However, since the Daya Bay, Ling Ao I and Ling Ao II complexes, like RENO, lie along a line, it is possible to evade this interference effect if the detector is roughly orthogonal to the line. The price of this choice is that one cannot benefit from the flux at the proposed HuiDong reactor complex, which would no longer be equidistant from the detector.

Our simulation also includes the flux from many distant reactors, of which TaiShan and YangJiang will begin commercial operations next year. We will see that these backgrounds have a nonnegligible effect on the probability of determination of the neutrino mass hierarchy and as a result they decrease the optimal baseline. They provide more background but also potentially more usable information for the determination of $\theta_{12}$.

\section{Data Analysis}

\subsection{Fourier Analysis and Reactor Flux Models}

In this note we will not consider a peak energy analysis [16] of the data, although this requires an understanding of the nonlinear response of the detector only at the locations of any two peaks, where it may be calibrated using radioactive decays. Instead we will consider a Fourier analysis [14], which has the advantage that it combines neutrinos from multiple peaks and so requires less flux, despite the fact that it requires an unprecedented understanding of the nonlinear response of the detector [17] over a wide range of energies. Following Refs. $[13,15]$ we will decompose this transform into a real and a complex part

$$
\begin{aligned}
& F_{c}^{i}(k)=\sum_{j} w^{i}\left(E_{j}\right) N\left(E_{j}\right) \cos \left(\frac{k L}{E_{j}}\right) \\
& F_{s}^{i}(k)=\sum_{j} w^{i}\left(E_{j}\right) N\left(E_{j}\right) \sin \left(\frac{k L}{E_{j}}\right)
\end{aligned}
$$

where we have summed over energy bins $j$, each centered at energy $E_{j}$ with $N\left(E_{j}\right)$ neutrinos observed. We use $1 \mathrm{keV}$ bins, but we have found that for such small bins our results are independent of the bin size. The index $i$ labels different Fourier transforms obtained using different weights $w^{i}(E)$. We will now explain the energy-dependent weight $w^{i}(E)$, which was introduced in Ref. [18].

We will be interested in the form of the Fourier transforms near the 1-3 oscillation peak $k=\left|\Delta M_{31}^{2}\right| / 2$. At these wavenumbers it is commonly believed that gross features of the spectrum are independent of the reactor flux and $P_{12}$, but in the case of a trivial weighting 
$w(E)=1$ this is not quite true. Recently Ref. [17] found that the quantities (3.3) used in the analysis of Refs. $[13,15]$ depend strongly upon the reactor flux model used and for some flux models even upon $\left|\Delta M_{32}^{2}\right|$, with variations as large as a factor of 5. In Ref. [18] it was shown that the dependence upon the reactor flux model and $\left|\Delta M_{32}^{2}\right|$ results from a dependence of the unweighted Fourier transform upon the high energy tail $(E>8 \mathrm{MeV})$ of the neutrino spectrum. As the high energy spectrum is only sensitive to $\Delta M_{\mathrm{eff}}^{2}$ and therefore not to the hierarchy $[9,16]$, this dependence introduces a kind of nuisance parameter, impeding the discovery of the hierarchy using the unweighted transform. In Ref. [18] it was shown that there exist weight functions $w(E)$ which gradually cut off the sensitivity to the high energy spectrum and so lead to a weighted Fourier transform without this spurious dependence. Furthermore it was shown that the weight functions actually improve the sensitivity of the Fourier transform to the hierarchy.

Once the high energy dependence is eliminated, the sensitivity of the weighted Fourier transform to the reactor flux model becomes negligible. Therefore, to maximize the compatibility of our analysis with that of Refs. [13, 15], we will use the Gaussian fit to the old reactor flux model of Ref. [25]. We will consider a general weight function

$$
w^{i}(E)=a_{1}{ }^{i} e^{-0.08\left(\frac{E}{\mathrm{MeV}}\right)^{2}}+a_{2}{ }^{i} e^{-0.08\left(\frac{E}{\mathrm{MeV}}-3.6\right)^{2}}+a_{3}{ }^{i} e^{-\frac{E}{3 \mathrm{MeV}}}+a_{4}{ }^{i} e^{-\frac{E}{6 \mathrm{MeV}}}+a_{5}{ }^{i} e^{-\left(\frac{E}{\mathrm{MeV}}-5.25\right)^{2} / 4} .
$$

For the $i$ th observable, the coefficients $a_{j}{ }^{i}$ will be optimized by a neural network as described in Subsec. 3.3. The weight functions have been chosen so as to fall off fast enough at high energy that the spurious effect of high energy neutrinos on the determination of the hierarchy will be negligible. They have also been chosen so as to allow the neural network to dynamically determine which part of the spectrum is the most important for a given observable. However the basis of weights itself has not been optimized. Once the basis has been optimized for a given experimental configuration, the chance of successfully determining the hierarchy will improve.

\subsection{Observables}

Now that we have inserted a scale factor into the Fourier transform, the qualitative features of the transformed spectrum depend primarily upon $P_{13}$, which gives a symmetric $F_{c}(k)$ with a central peak and damped oscillations with a wavenumber of order $L\langle 1 / E\rangle$ where $\langle 1 / E\rangle$ is the average value of $1 / E$. At medium baselines, of order $40-80 \mathrm{~km}$, this wavenumber is of order $\Delta M_{21}^{2}$. Similarly the $P_{13}$ contribution to the sine transform $F_{c}(k)$ is antisymmetric about $k=\left|\Delta M_{31}^{2}\right| / 2$, with a maximum at higher $k$ and a minimum at lower $k$. Again, as one varies $k$ from $k=\left|\Delta M_{31}^{2}\right| / 2$ one finds a series of ever shrinking oscillations separated 
by a characteristic distance of order $\Delta M_{21}^{2}$. At $L=58 \mathrm{~km}$ analytic approximations to these features are derived in Ref. [16].

As the Fourier transforms are linear, they add the transform of the $P_{23}$ oscillations to that of the larger $P_{13}$ oscillations. The hierarchy can be determined from the way in which the resulting asymmetric perturbation breaks the (anti)symmetry of the (sine) cosine transform of $P_{13}$. It was observed in Ref. [13] and derived in Ref. [16] that in the case of the normal (inverted) hierarchy the $P_{23}$ oscillations render the first minimum $R$ to the right of the global maximum of $F_{c}$ larger (smaller) than its mirror image $L$ and similarly render the maximum $P$ of $F_{s}$ just to the right of $k=\left|\Delta M_{31}^{2}\right| / 2$ larger (smaller) than the minimum just to its left $V$. Ref [13] introduced two parameters which characterize these effects

$$
p_{1}=R L=\frac{R-L}{R+L}, \quad p_{2}=P V=\frac{P-V}{P+V}
$$

finding that positive (negative) values of these two parameters tend to indicate the normal (inverted) hierarchy. We introduce the additional notation $p_{1}$ and $p_{2}$ to simplify the equations that follow.

In Ref. [16] two more parameters were suggested. First, the value $\phi$ of $F_{s}$ at the maximum of $F_{c}$, which is positive (negative) for the normal (inverted) hierarchy. Second, the difference in values $m_{ \pm}$of the maxima of hierarchy-dependent nonlinear Fourier transforms

$$
F_{n}^{ \pm}(k)=\sum_{j} w^{i}\left(E_{j}\right) N\left(E_{j}\right) \cos \left(k \frac{L}{E_{j}} \pm 2 \pi \alpha\left(\frac{k}{2 \pi} \frac{L}{E_{j}}\right)\right) .
$$

These can be encoded into the normalized observables

$$
p_{3}=\frac{\phi}{P+V}, \quad p_{4}=\frac{m_{+}-m_{-}}{m_{-}+m_{+}} .
$$

We have repeated our analysis using a new parameter $p_{5}$ which is defined identically to $p_{3}$ except that it is evaluated at the maximum of the norm $\left(F_{c}^{2}+F_{s}^{2}\right)$ of the Fourier transform and not at the maximum of $F_{c}$ alone. The resulting improvement in our determination of the hierarchy is within our statistical errors and so we have not included it in our results below.

\subsection{Neural Network}

In Subsec. 3.2 we identified four quantities $p_{i}$ which may be used to determine the hierarchy. The sign of any one is already a good indicator of the hierarchy, in fact they are reasonably degenerate. 
Our goal is to determine the best indicator, that which, given a dataset, has the highest probability of correctly determining the mass hierarchy. As these indicators are not precisely degenerate, the best indicator will not be a single $p_{i}$ but rather some combination of all 4 . For simplicity we will consider linear combinations

$$
I=b_{0}+\sum_{i=1}^{4} b_{i} p_{i}
$$

where $b_{i}$ are 5 constants. To each of these observables $p_{i}$ except for the constant $i=0$ we associate a weight function $w^{i}(E)$ which is determined by the 5 constants $a_{j}{ }^{i}$ defined in Eq. (3.2). A different weight may be optimal for each observable $p_{i}$, thus in all we need to optimize 20 constants $a_{j}{ }^{i}$ and 5 constants $b_{i}$. The optimal values are those such that, given a set of experiments in which the neutrino mass hierarchy is normal in as many as it is inverted ${ }^{1}$, the chance of success is the highest. The chance of success is defined to be the average of the percentage of normal hierarchy experiments for which $I$ is positive with the number of inverted hierarchy experiments for which $I$ is negative. Clearly the overall scale of $b_{i}$ is irrelevant, but the optimal choice of $b_{i}$ in general depends on the baseline, the reactors that are operational, the geometry of the reactors with respect to the detector and even the time for which the experiment has run.

We will optimize these coefficients using a 2 layer neural network. The first layer, for each $p_{i}$, will optimize the weight coefficients $a_{j}{ }^{i}$ so as to maximize the number of experiments in which $p_{i}$ alone determines the hierarchy. The second layer, as described above, then optimizes the weights $b_{i}$ of the weighted $p_{i}$ 's. As we will have fit 44 coefficients one may worry about overfitting if the number of experiments is too small. Therefore we have only reported the results of the full 2 layer neural network in the case of BaiYunZhang, as that is the only site for which we have simulated 4000 experiments. For this site overfitting effects appear to affect the probability of success by less than $1 \%$. To be sure that our numbers are not inflated by overfitting, in every case in which we quote the result of a neural network the data used to test the chance of success has no overlap with the data used to train the neural network.

Summarizing, for each value of $i$ from 1 to 4 the first layer of the neural network optimizes $a_{j}{ }^{i}$ of Eq. (3.2) so as to maximize the probability of success of $p_{i}$ alone. Then the second layer optimizes the $b_{i}$ in Eq. (3.6) so as to maximize the probability of success of the indicator $I$.

This procedure is somewhat different from the usual approach in which the coefficients at

\footnotetext{
${ }^{1}$ This corresponds to a Bayesian prior which assigns a $50 \%$ chance to each hierarchy. To use the hierarchy confidence that may be obtained at $\mathrm{NO} \nu \mathrm{A}$ or potentially $\mathrm{T} 2 \mathrm{~K}$ one need only match the ratio of hierarchies in the simulated experiments.
} 
all of the different layers are optimized simultaneously. The reason for this difference is the very high level of degeneracy between our 44 parameters. Indeed, we will see in the following subsection that any one of these parameters individually already yields a determination of the hierarchy which is qualitatively similar to that of the network. This is not to say that the network is unimportant in the planning of these experiments, on the contrary we will see in Subsec. 4.1 that it favors a nonlinear Fourier transform heavy analysis at shorter baselines which results in an optimal baseline which is several kilometers shorter than that preferred by a classical $R L+P V$ analysis. However the degeneracy does have two important consequences.

First of all, since the individual $p_{i}$ 's are largely degenerate, weights that optimize individual $p_{i}$ 's are also nearly optimal for $I$ itself. This means that the weights $a_{j}{ }^{i}$ which optimize $p_{i}$ will approximately optimize $I$. Second it implies that there are number of degenerate combinations of the fitting parameters. If all of the parameters are optimized simultaneously, as is usual in a feedforward neural network, then to find the optimal parameters our algorithm would use a gradient decent in a 44-dimensional space. However, as most of the directions are degenerate and we only have 4,000 data points, convergence would be slow and the process would be dominated by statistical errors. In addition, by fitting $I$ directly overfitting would lead to a larger bias in the coefficients.

We have checked, in 3 applications of our 2 layer neural network, that under and overfitting affect the efficiency less than statistical errors when 4000 experiments are used as a training set, although not necessarily when only 2000 are used. To perform this check we have compared an upper and lower bound on the neural network chance of success. The upper bound is determined by simply finding the best weights for all of the simulated data and applying these weights to the same simulated data. The lower bound is obtained by dividing the simulated data into 4 subsets, training on three subsets and then applying the resulting weights to the other subset. This procedure is repeated four times, with each subset used once as the target, and the results are averaged. The upper and lower bounds were separated in every 4000 simulation case by less than 1 percent.

\subsection{The Neural Network Applied to BaiYunZhang}

We will now illustrate the functioning of the neural network on 60 kton years of neutrino detection underneath the mountain BaiYunZhang, described in Table 5. We will assume that all of the reactors described in Table 4 are operational. This is an overly pessimistic assumption, as Daya Bay II is scheduled for completion in 2020 [12] whereas the reactors at HuiDong and Lufeng are still awaiting final approval. Once construction begins, an 


\begin{tabular}{c|l|l|l|l|l|l} 
Weight & $p_{1}$ & $p_{2}$ & $p_{3}$ & $p_{4}$ & $p_{1}+p_{2}$ & NN Level 2 \\
\hline \hline 1 & $74.6 \%$ & $76.9 \%$ & $74.9 \%$ & $73.0 \%$ & $77.5 \%$ & \\
\hline$e^{-0.08\left(\frac{E}{\mathrm{MeV}}\right)^{2}}$ & $75.7 \%$ & $77.0 \%$ & $74.5 \%$ & $72.6 \%$ & $77.6 \%$ & \\
\hline$e^{-0.08\left(\frac{E}{\mathrm{MeV}}-3.6\right)^{2}}$ & $74.9 \%$ & $77.0 \%$ & $74.8 \%$ & $73.0 \%$ & $77.3 \%$ & \\
\hline$e^{-E /(3 \mathrm{MeV})}$ & $75.5 \%$ & $74.1 \%$ & $71.6 \%$ & $69.4 \%$ & $75.8 \%$ & \\
\hline$e^{-E /(6 \mathrm{MeV})}$ & $76.2 \%$ & $76.1 \%$ & $73.4 \%$ & $71.4 \%$ & $71.6 \%$ & \\
\hline$e^{-\left(\frac{E}{\mathrm{MeV}}-5.25\right)^{2} / 4}$ & $70.8 \%$ & $74.2 \%$ & $72.4 \%$ & $77.4 \%$ & $72.3 \%$ & \\
\hline $\mathrm{NN} \mathrm{level} \mathrm{1}$ & $75.7 \%$ & $77.6 \%$ & $76.3 \%$ & $77.6 \%$ & & $78.0 \%$ \\
\hline
\end{tabular}

Table 1: The probability of successfully determining the hierarchy at a 20 kton detector under BaiYunZhang after 3 years of running using various indicators and layers of the neural network independently. It is assumed that all planned reactors have been built. The bottom right percentage is our final answer, it does not suffer from overfitting as the neural network is not trained on the same data to which it is applied. The other entries are the average chances of success of 2000 experiments with each hierarchy.

individual reactor generally takes about four and a half years to build in China, and generally at a complex of reactors, construction on one reactor begins each year. Therefore, unless this scheduling changes, one may expect HuiDong and LuFeng to take 10 years from the beginning of construction to reach full capacity, and thus not to be at full capacity before data taking begins at Daya Bay II.

We have considered four sets of 1000 experiments, each of which contains 500 with each hierarchy. The neural network is trained separately on each subset of three sets and then tested on the remaining set. The final result for the probability of success, displayed in the bottom right corner of Table 1 is the average of the four probabilities of success obtained from the testing of the neural network on each set. Notice that while the nonlinear Fourier transform $p_{4}$ and the high energy weight $e^{-\left(\frac{E}{\mathrm{MeV}}-5.25\right)^{2} / 4}$ in general perform poorly, the high energy nonlinear transform alone produces a $77.4 \%$ chance of success, which is the highest of any individual observable with any individual weight. Thus the nonlinear Fourier transform performs better at high energies, whereas the linear Fourier transform performs better at low energies. As the oscillation probability depends on $L / E$, this will imply that the nonlinear transform is optimized at shorter baselines than the linear transform methods, which is why we obtain a shorter optimal baseline than was obtained in Refs. [15, 10].

While the neural network does outperform $p_{1}+p_{2}$ with any weight, this effect is quite small. When we turn to simulations with a single baseline in Subsec. 4.1 we will see that at such short baselines even the second layer of the neural network alone significantly outper- 


\begin{tabular}{l|l|l|l} 
Baseline & $N_{\bar{\nu}_{e}}$ at $60 \mathrm{ky}$ & Hierarchy at 60 ky & Hierarchy at 120 ky \\
\hline \hline $42 \mathrm{~km}$ & 64,860 & $86.0 \%(81.9 \%)$ & $94.1 \%(90.3 \%)$ \\
\hline $44 \mathrm{~km}$ & 55,578 & $86.4 \%(83.3 \%)$ & $95.1 \%(90.3 \%)$ \\
\hline $46 \mathrm{~km}$ & 48,030 & $87.7 \%(84.9 \%)$ & $95.5 \%(93.1 \%)$ \\
\hline $48 \mathrm{~km}$ & 41,900 & $88.4 \%(86.2 \%)$ & $95.5 \%(93.2 \%)$ \\
\hline $50 \mathrm{~km}$ & 36,931 & $88.7 \%(87.1 \%)$ & $95.4 \%(94.2 \%)$ \\
\hline $52 \mathrm{~km}$ & 32,915 & $88.1 \%(86.4 \%)$ & $95.0 \%(92.9 \%)$ \\
\hline $54 \mathrm{~km}$ & 29,679 & $86.4 \%(85.8 \%)$ & $94.8 \%(93.9 \%)$ \\
\hline $56 \mathrm{~km}$ & 27,080 & $86.8 \%(85.5 \%)$ & $93.7 \%(93.2 \%)$ \\
\hline $58 \mathrm{~km}$ & 25,000 & $84.3 \%(84.0 \%)$ & $94.1 \%(93.4 \%)$ \\
\hline $60 \mathrm{~km}$ & 23,342 & $81.9 \%(81.8 \%)$ & $93.2 \%(92.4 \%)$ \\
\hline
\end{tabular}

Table 2: The observed $\bar{\nu}_{e}$ flux from a single $17.4 \mathrm{GW}$ reactor and the probability of determining the hierarchy as a function of baseline and number of kton years determined using the second layer of the neural network. The neural networks trained on the neighboring baselines, and the values of the coefficients $b_{i}$ used to determine the chance of success are the averages of the coefficients at the available neighboring baselines. Probabilities in parenthesis are obtained using $p_{1}+p_{2}$ as in Ref. [13].

forms $p_{1}+p_{2}$. However this improvement is reduced when multiple baselines are present. Of course an optimization of the basis of weights and a longer running of the neural network, trained on more data, will enhance its performance.

\section{Optimizing the Baseline and Geometry}

\subsection{The Optimal Baseline}

As a first application of our simulation we have attempted to determine the optimal baseline in an idealized situation in which all $17.4 \mathrm{GW}$ of thermal capacity are located at a single reactor, at a fixed baseline. This eliminates the interference effects of Ref. [16] which will be investigated in Subsec. 4.2. The probability of success then depends only upon the baseline, the number of ktons of the detector multiplied by the number of years of observation and the method of data analysis. We will consider an unweighted Fourier transform analysis with two indicators, $p_{1}+p_{2}$ which was introduced in Ref. [13] and also the second layer of our neural network. The results, considering 2000 experiments of each hierarchy, are displayed in Table 2. 


\begin{tabular}{l|l|l|l|l|l|l|l}
$L$ & $\mathrm{ky}$ & $0^{\circ}$ & $15^{\circ}$ & $30^{\circ}$ & $45^{\circ}$ & $60^{\circ}$ & $75^{\circ}$ \\
\hline \hline 50 & 60 & $66.3(66.4)$ & $66.4(66.5)$ & $70.6(70.7)$ & $76.4(75.9)$ & $82.0(81.5)$ & $85.8(84.7)$ \\
\hline 50 & 120 & $73.5(72.1)$ & $72.1(71.8)$ & $78.3(76.5)$ & $85.1(84.0)$ & $90.8(89.7)$ & $93.9(91.7)$ \\
\hline 58 & 60 & $63.6(64.2)$ & $63.8(64.4)$ & $67.9(67.6)$ & $73.8(73.7)$ & $79.8(78.9)$ & $83.3(82.4)$ \\
\hline 58 & 120 & $73.0(71.6)$ & $74.2(72.4)$ & $77.5(75.7)$ & $84.9(83.5)$ & $89.5(88.2)$ & $93.0(91.5)$ \\
\hline
\end{tabular}

Table 3: The probability (\%) of determining the hierarchy as a function of baseline in kilometers and number of kton years determined using unweighted Fourier transforms and only the second layer of the neural network. The neural network is trained on the neighboring angles. The value in parenthesis is the percentage chance of success with only $p_{1}+p_{2}$. The baseline is the distance from the center of mass of the Daya Bay, Ling Ao I and II reactor complexes considering the distances between these complexes. The angles are measured with respect to the line that nearly passes through all three complexes.

\subsection{Interference Between Reactors in the Same Complex}

No single reactor produces enough flux to determine the neutrino hierarchy in a reasonable amount of time. Thus it is inevitable that a complex of reactors needs to be used, and often the distances between these reactors is considerable. For example in Ref. [19] the proposed site for Daya Bay II is $3.5 \mathrm{~km}$ closer to the reactor complexes at Daya Bay and Ling Ao than to that planned at HuiDong. These different baselines mean that for some energies the neutrinos from one reactor will arrive at the 1-3 oscillation maximum while neutrinos from the other will arrive at the 1-3 oscillation minimum, greatly reducing the amplitude of the 1-3 oscillations whose observation is necessary to determine the hierarchy [16]. Note that the neutrinos arriving from different reactors are not coherent, the interference effect results from the addition of probabilities and not wavefunctions.

Such an interference effect is present using the reactors at Daya Bay and Ling Ao alone, because Daya Bay and Ling Ao I are separated by $1.1 \mathrm{~km}$ and Ling Ao II is 500 meters further. Fortunately these reactors all lie more or less along a line, so a medium baseline detector perpendicular to this line will be the same distance from each reactor, eliminating the interference effect. In Table 3 we have determined the effect of this interference on the probability of success for a detector as a function of its distance from the center of mass of Daya Bay and Ling Ao, its angle with respect to this line and the number kton years of observations. To illustrate the effect of the angle, the interference from other, more distant, reactors is not considered. These will be included in the full simulations reported in Sec. 5 . 


\begin{tabular}{l|l|l|l|l} 
Name & Status & Latitude & Longitude & Thermal Power \\
\hline \hline Daya Bay & Operational & $22^{\circ} 35^{\prime} 53^{\prime \prime} \mathrm{N}$ & $114^{\circ} 32^{\prime} 35^{\prime \prime} \mathrm{E}$ & $5.8 \mathrm{GW}$ \\
\hline Ling Ao I & Operational & $22^{\circ} 36^{\prime} 19^{\prime \prime} \mathrm{N}$ & $114^{\circ} 33^{\prime} 4^{\prime \prime} \mathrm{E}$ & $5.8 \mathrm{GW}$ \\
\hline Ling Ao II & Operational & $2^{\circ} 36^{\prime} 31^{\prime \prime} \mathrm{N}$ & $114^{\circ} 33^{\prime} 14^{\prime \prime} \mathrm{E}$ & $5.8 \mathrm{GW}$ \\
\hline TaiShan I & Under Construction & $21^{\circ} 55^{\prime} 9^{\prime \prime} \mathrm{N}$ & $112^{\circ} 58^{\prime} 57^{\prime \prime} \mathrm{E}$ & $9.2 \mathrm{GW}$ \\
\hline TaiShan II & Planned & $21^{\circ} 55^{\prime} \mathrm{N}$ & $112^{\circ} 59^{\prime} \mathrm{E}$ & $9.2 \mathrm{GW}$ \\
\hline YangJiang I & Under Construction & $21^{\circ} 42^{\prime} 29^{\prime \prime} \mathrm{N}$ & $112^{\circ} 15^{\prime} 32^{\prime \prime} \mathrm{E}$ & $5.8 \mathrm{GW}$ \\
\hline YangJiang II & Under Construction & $21^{\circ} 42^{\prime} 36^{\prime \prime} \mathrm{N}$ & $112^{\circ} 15^{\prime} 41^{\prime \prime} \mathrm{E}$ & $5.8 \mathrm{GW}$ \\
\hline YangJiang III & Planned & $21^{\circ} 43^{\prime} \mathrm{N}$ & $112^{\circ} 16^{\prime} \mathrm{E}$ & $5.8 \mathrm{GW}$ \\
\hline HuiDong & Planned & $22^{\circ} 42^{\prime} \mathrm{N}$ & $115^{\circ} 0^{\prime} \mathrm{E}$ & $17.4 \mathrm{GW}$ \\
\hline LuFeng & Planned & $22^{\circ} 45^{\prime} \mathrm{N}$ & $115^{\circ} 49^{\prime} \mathrm{E}$ & $17.4 \mathrm{GW}$ \\
\hline
\end{tabular}

Table 4: Reactors in Guangdong

\section{Comparing Detector Locations Near Daya Bay}

\subsection{Locations of Reactors and Detectors}

China's Guangdong province is among the best locations for a medium baseline reactor experiment because it contains a powerful reactor complex consisting of 2 reactors at Daya Bay and 4 at Ling Ao and yet it is free from the large reactor neutrino backgrounds caused by many smaller complexes in France and, modulo tsunami induced shutdowns, in Japan. In the next few years a number of new reactor complexes will be completed in Guangdong. Two distant complexes, TaiShan and YangJiang, will see their first reactors generate power already next year. The TaiShan reactors will be the world's first completed EPR reactors, which is an advantage as it will mean more than $50 \%$ more neutrino flux per reactor than the other reactors in Guangdong. On the other hand, it means that there may be some deviation between its spectrum and that measured at Daya Bay. Two closer complexes, HuiDong and LuFeng, have already passed several critical steps in the approval process and may be built. The relevant reactors are listed in Table 4.

Depending on the detector location the new reactors may help or hinder the determination of the neutrino hierarchy. They will help at each detector which is equidistant from two reactors, as the fluxes add without interference. This is the case for the proposed location of Ref. [12] and also at our proposed location DongKeng. If the two reactors are nearly at the same distance, as in the proposal of Ref. [19] and our proposed location HuangDeDing, the addition of the second reactor will increase the flux but interference between the neutrinos from different reactor complexes will decrease the amplitudes of the 1-3 oscillations. 


\begin{tabular}{l|l|l|l|l|l|l|l|l} 
Name & Altitude & Latitude (N) & Longitude (E) & DB & TS & YJ & HD & LF \\
\hline \hline BaiYunZhang & $1000 \mathrm{~m}$ & $2^{\circ} 53^{\prime} 52^{\prime \prime}$ & $114^{\circ} 15^{\prime} 14^{\prime \prime}$ & 44.5 & 170.5 & 244.1 & 79.5 & 161.6 \\
\hline ShiYaTou & $500 \mathrm{~m}$ & $2^{\circ} 52^{\prime} 14^{\prime \prime}$ & $114^{\circ} 17^{\prime} 28^{\prime \prime}$ & 39.7 & 171.6 & 245.7 & 75.5 & 157.6 \\
\hline ShuangFeiJi & $700 \mathrm{~m}$ & $2^{\circ} 54^{\prime} 19^{\prime \prime}$ & $114^{\circ} 10^{\prime} 0^{\prime \prime}$ & 51.6 & 164.2 & 237.0 & 88.3 & 170.6 \\
\hline SanJiaBi & $600 \mathrm{~m}$ & $2^{\circ} 54^{\prime} 8^{\prime \prime}$ & $114^{\circ} 10^{\prime} 41^{\prime \prime}$ & 50.6 & 164.9 & 237.8 & 87.3 & 171.6 \\
\hline XiangTouShan & $800 \mathrm{~m}$ & $23^{\circ} 15^{\prime} 24^{\prime \prime}$ & $114^{\circ} 21^{\prime} 0^{\prime \prime}$ & 75.4 & 205.0 & 275.3 & 90.4 & 160.9 \\
\hline BaiMianShi & $400 \mathrm{~m}$ & $23^{\circ} 6^{\prime} 27^{\prime \prime}$ & $114^{\circ} 37^{\prime} 2^{\prime \prime}$ & 56.8 & 214.1 & 288.0 & 60.3 & 129.7 \\
\hline DongKeng & $200 \mathrm{~m}$ & $22^{\circ} 6^{\prime} 4^{\prime \prime}$ & $112^{\circ} 31^{\prime} 9^{\prime \prime}$ & 216.5 & 51.8 & 51.0 & 264.1 & 347.3 \\
\hline HuangDeDing & $500 \mathrm{~m}$ & $22^{\circ} 5^{\prime} 23^{\prime \prime}$ & $112^{\circ} 29^{\prime} 55^{\prime \prime}$ & 218.9 & 53.3 & 48.8 & 266.5 & 349.7 \\
\hline
\end{tabular}

Table 5: Potential locations for Daya Bay II detectors and distances to reactors in $\mathrm{km}$. DB indicates the distance to the weighted center of Daya Bay and Ling Ao I and II.

However in general the additional reactors will be so distant that the 1-3 oscillation peaks will be too close together to be distinguishable by a detector with resolution $\sigma_{E}$. As a result, the additional reactors will simply supply a background which impedes the measurement of the hierarchy.

A medium baseline detector experiment may also be used to measure $\theta_{12}$. This can be done by comparing the flux at the $1-2$ oscillation minima and maxima. If the new reactor is twice as far away as the desired reactor, as is the case for most of the positions that we will consider, then the distant reactor will be at its 1-2 minimum at the same energy range in which neutrinos from the near reactor arrive at their 1-2 maximum. This means that at the 1-2 maximum, where sensitivity to the hierarchy and to $\theta_{12}$ is maximized, the neutrino flux will be dominated by noise from the distant reactor [16]. In general this makes the determination of both the hierarchy and $\theta_{12}$ more difficult. However the 1-2 oscillations of distant reactors do lie within the resolution of the detector and so these can be used to gain more information about $\theta_{12}$. Furthermore, if multiple detectors are considered, then they can break the degeneracy between $\theta_{12}$ and flux from distant reactors, allowing a more precise determination of both.

In Table 5 we list the detector positions that we propose together with BaiMianShi, which was proposed in Ref. [19]. These points are chosen to be at a medium baseline, to minimize interference effects, to be underneath mountains to minimize backgrounds and when possible to be a similar distance from multiple reactors to enhance the useful neutrino flux. No site that we have found simultaneously achieves all of these goals. 


\subsection{Monte Carlo Results}

The simulation of planned reactors is impeded by the fact that we do not know the configurations of the reactors. These configurations are important when the reactors are used as sources of flux because it determines the reduction in the resulting 1-3 peaks due to interference. It is not important when the reactors provide a background source. While in general these configurations cannot be predicted, one natural guess is that the reactors will follow the coast, which suggests for example that interference will not pose a problem for neutrinos generated at TaiShan. However we have made no such assumptions in our analysis. In Tables 6 and 7 we present our 60 kton year and 120 kton year results respectively. We use a Roman numeral I to signify assumed interference at unbuilt reactors equal to that of Daya Bay and Ling Ao as observed at BaiMianShi and a Roman number II to signify no interference at unbuilt reactors.

Note that the sites with the highest percentage of success, BMS Ideal and DongKeng, have the smallest mountains. This is because both are equidistant from two reactor complexes so as to benefit from the increased flux, but there is no peak at that location. Instead they are under the highest equidistant point, which is between 200 and 300 meters in both cases. The peaks of the corresponding mountains, BaiMianShi and XiKengDing, are each over 500 meters high but as can be seen from the tables, the interference effects are so great that these do not allow accurate determinations of the hierarchy.

Instead the best locations for a neutrino detector lie under the mountains orthogonal to the line between Daya Bay and Ling Ao. A number of mountains between 44 and 51 $\mathrm{km}$ offer similar chances of determining the hierarchy. In general the closest and highest, BaiYunZhang, is the best. However if the planned reactors at HuiDong and LuFeng are not built, then the more distant mountains such as SanJiaBi and ShuangFeiJi gain a clear advantage. Use of the full, 2-layer, neural network leads to a modest improvement in cases with large backgrounds with many reactors. For example, if all proposed reactors are built, then with 60 kton years the full neural network yields a $78.0 \%$ chance of success at BaiYunZhang and SanJiaBi and $76.5 \%$ at ShuangFeiJi, representing on average a $1 \%$ improvement over an analysis based on $p_{1}+p_{2}$ alone. The statistical errors in these chances of success are slightly less than 1\%. Indeed, one expects that with more data the chances of success at SanJiaBi and ShuangFeiJi will converge. SanJiaBi and ShuangFeiJi are in the middle of a wilderness reserve and so may not provide suitable sites. However, $3 \mathrm{~km}$ to their south, at the edge of the reserve, and at the same baseline from Daya Bay is the 800 meter peak of YinPingShan, which we expect to yield a similar chance of success. For example, we have measured an 800 meter elevation at the ZiYanGe pagoda at $22^{\circ} 53^{\prime} 18^{\prime \prime} \mathrm{N}$ and $114^{\circ} 8^{\prime} 43^{\prime \prime}$ E. Slightly longer 


\begin{tabular}{l|l|l|l|l|l|l} 
Name & Simulations & All & No LF & No HD & No HD/LF & DB and LA \\
\hline \hline BaiYunZhang & 4000 & $77.6 \%$ & $77.9 \%$ & $81.0 \%$ & $82.4 \%$ & $83.6 \%$ \\
\hline SYT+XTS & 2000 & $71.5 \%$ & $73.0 \%$ & $75.8 \%$ & $76.4 \%$ & $78.3 \%$ \\
\hline SanJiaBi & 2000 & $77.5 \%$ & $77.9 \%$ & $81.1 \%$ & $82.6 \%$ & $85.8 \%$ \\
\hline ShuangFeiJi & 2000 & $74.6 \%$ & $76.7 \%$ & $80.3 \%$ & $81.7 \%$ & $85.2 \%$ \\
\hline BaiMianShi I & 2000 & $53.0 \%$ & $51.7 \%$ & $65.3 \%$ & $68.1 \%$ & $72.2 \%$ \\
\hline BaiMianShi II & 2000 & $49.3 \%$ & $48.9 \%$ & $64.6 \%$ & $66.7 \%$ & $69.8 \%$ \\
\hline BMS Ideal I & 2000 & $74.4 \%$ & $78.3 \%$ & $62.9 \%$ & $66.5 \%$ & $68.9 \%$ \\
\hline BMS Ideal II & 2000 & $86.4 \%$ & $88.2 \%$ & $62.9 \%$ & $67.2 \%$ & $70.3 \%$ \\
\hline DongKeng I & 2000 & $74.9 \%$ & $74.5 \%$ & $74.6 \%$ & $75.2 \%$ & $49.3 \%$ \\
\hline DongKeng II & 2000 & $87.8 \%$ & $87.9 \%$ & $88.2 \%$ & $88.4 \%$ & $50.8 \%$ \\
\hline HuangDeDing I & 2000 & $58.5 \%$ & $58.7 \%$ & $58.8 \%$ & $59.2 \%$ & $50.1 \%$ \\
\hline HuangDeDing II & 2000 & $63.7 \%$ & $63.6 \%$ & $65.0 \%$ & $64.4 \%$ & $50.8 \%$ \\
\hline
\end{tabular}

Table 6: Potential locations for Daya Bay II detectors and chances of success using $p_{1}+p_{2}$ for 60 kton years of flux and choices of active reactors. The first column considers all planned reactors. In the second LuFeng is excluded, in the third HuiDong is instead excluded, in the fourth both are excluded and in the last only Daya Bay and Ling Ao are considered. SYT + XTS refers to two detectors, one under ShiYiTou and one under XiangTouShan, each of which is half as large as indicated in the corresponding column, simply summing the $L / E$ spectra. BMS ideal is a 200 meter high point $2 \mathrm{~km}$ from BaiMianShi which is equidistant from the center of mass of the Daya Bay/Ling Ao complex and HuiDong. Both BaiMianShi I and BMS Ideal I assume that the baselines to the HuiDong reactors will differ by as much as those to Daya Bay and Ling Ao reactors, whereas II assume that the HuiDong reactors are coincident. Similarly DongKeng and XiKengDing I (II) assume that there is (not) interference at TaiShan and YangJiang. 


\begin{tabular}{l|l|l|l|l|l|l} 
Name & Simulations & All & No LF & No HD & No HD/LF & DB and LA \\
\hline \hline BaiYunZhang & 2000 & $87.1 \%$ & $87.4 \%$ & $88.8 \%$ & $90.7 \%$ & $91.8 \%$ \\
\hline SYT+XTS & 1000 & $80.3 \%$ & $81.9 \%$ & $84.0 \%$ & $85.2 \%$ & $87.3 \%$ \\
\hline SanJiaBi & 1000 & $85.8 \%$ & $86.5 \%$ & $89.5 \%$ & $91.8 \%$ & $93.4 \%$ \\
\hline ShuangFeiJi & 1000 & $84.4 \%$ & $85.6 \%$ & $88.7 \%$ & $91.2 \%$ & $94.5 \%$ \\
\hline BaiMianShi I & 1000 & $55.3 \%$ & $55.7 \%$ & $73.7 \%$ & $78.8 \%$ & $80.7 \%$ \\
\hline BaiMianShi II & 1000 & $44.9 \%$ & $45.7 \%$ & $74.0 \%$ & $77.3 \%$ & $80.8 \%$ \\
\hline BMS Ideal I & 1000 & $84.9 \%$ & $88.3 \%$ & $72.4 \%$ & $78.1 \%$ & $79.2 \%$ \\
\hline BMS Ideal II & 1000 & $93.9 \%$ & $95.3 \%$ & $71.4 \%$ & $79.4 \%$ & $82.4 \%$ \\
\hline DongKeng I & 1000 & $83.8 \%$ & $83.3 \%$ & $84.0 \%$ & $83.7 \%$ & $49.7 \%$ \\
\hline DongKeng II & 1000 & $95.0 \%$ & $95.6 \%$ & $95.3 \%$ & $95.8 \%$ & $51.5 \%$ \\
\hline HuangDeDing I & 1000 & $62.3 \%$ & $61.3 \%$ & $63.4 \%$ & $62.7 \%$ & $51.5 \%$ \\
\hline HuangDeDing II & 1000 & $72.7 \%$ & $71.8 \%$ & $72.7 \%$ & $72.6 \%$ & $50.6 \%$ \\
\hline
\end{tabular}

Table 7: Potential locations for Daya Bay II detectors and chances of success using $p_{1}+p_{2}$ for 120 kton years of flux and choices of active reactors. Labels as in Table 6 .

baselines can be achieved using somewhat smaller mountains, for example we have found a 660 meter peak at $22^{\circ} 53^{\prime} 38^{\prime \prime} \mathrm{N}$ and $114^{\circ} 8^{\prime} 1^{\prime \prime}$ E. 2 kilometers further from Daya Bay lie the 500 meter hills of GuanYin park.

However even a $2 \sigma$ determination in Guangdong appears to require more than 120 kton years $^{2}$. As a result, either multiple detectors or a detector larger than 20 ktons may be preferable. Multiple detectors are preferable for breaking the degeneracy between $\theta_{12}$ and the unknown neutrino flux from distant reactors [16].

\section{Conclusions}

We have simulated medium baseline reactor neutrino experiments with a single baseline and also at real positions in Guangdong province. We found that in the presence of a single reactor the optimal baseline is about $48-52 \mathrm{~km}$. The presence of multiple reactors and so multiple baselines has a number of implications. First of all, if the difference between baselines is between 1 and $5 \mathrm{~km}$ then interference effects greatly reduce the chance of determining the hierarchy, although as the location BMS ideal shows, this loss can be more than offset

\footnotetext{
${ }^{2}$ The situation near the Yeonggwang reactor complex in South Korea is similar. To avoid interference a detector must be to the south southeast. But then one must choose between a 400 meter hill at a baseline of $47.4 \mathrm{~km}$ and Mudeungsan whose 950 meter peak is at $61.2 \mathrm{~km}$.
} 
by the presence of flux from an equidistant reactor complex. Unfortunately in Guangdong it is difficult to find a mountain which is equidistant from a pair of reactor complexes at a sufficiently short baseline to take advantage of this.

We have also seen that the presence of multiple reactors, even at distances in excess of $200 \mathrm{~km}$, significantly affects the chance of successfully determining the hierarchy at such an experiment. In a few years a number of reactors about $200 \mathrm{~km}$ from both Daya Bay and RENO will become operational. This loss can be somewhat compensated by reducing the baseline, thus adding flux from the desired reactors. As a result, the more reactors that will be built, the lower the optimal baseline. This means that, in many cases the optimal location for a medium baseline reactor neutrino experiment is BaiYunZhang, only $44.5 \mathrm{~km}$ from Daya Bay.

The biggest challenge facing such an experiment is a nonlinear systematic error in the determination of the prompt energy $\left(E_{e}=E_{\nu}-0.8 \mathrm{MeV}\right)$ from the number of photoelectrons observed in the photomultipliers. A successful determination of the hierarchy using the Fourier transform requires the observed 1-3 peaks to be periodic in $L / E$, so that they add constructively in the Fourier transform. Even a small systematic shift in the determination at the energy in some range of energies and at some locations in the detector can ruin this constructive interference, destroying the peak structure of the Fourier transform. It was determined in Ref. [17] that to determine the hierarchy using Fourier transform methods one requires an understanding of the nonlinear response of the detector an order of magnitude better than that achieved at KamLAND. In our study we have assumed that this nonlinear response is understood perfectly, and so our results yield upper bounds on the chance of successfully determining the hierarchy.

With multiple identical detectors at sufficiently different baselines, the hierarchy can be determined by comparing the locations of peaks that would be at the same energy in the absence of 2-3 oscillations. For example, the energy of the 10th peak at 40 kilometers will differ from that of the 15 th peak at 60 kilometers by 1 percent, and the sign of the difference determines the hierarchy. Since both of these peaks are at about the same energy, the nonlinear responses are expected to be the same and so do not affect the energy difference.

On the other hand, in the case of a single detector, the hierarchy can nonetheless be determined by measuring the energies of just two peaks in the untransformed spectrum [16], one at high energy which determines $\Delta M_{\text {eff }}^{2}[24,17,16]$ and one at low energy which removes the remaining degeneracy in the mass differences. The nonlinear response can be calibrated more precisely at certain discrete energies using radioactive decays which produce photons at corresponding energies. At such energies it is possible to understand the nonlinear response 
to a sufficient precision to determine the energy of the closest peaks, while at a sufficiently short baseline the peaks can simply be counted and identified to convert this energy into a combination of the mass differences as described in Ref. [16]. Therefore, with sufficient flux, an identification of individual peaks requires an understanding of the nonlinear detector response at only two energies, and not throughout the spectrum as is required by the Fourier transform method and in general the $\chi^{2}$ method. A precise determination of the hierarchy will rely on a combination of these methods and so a determination of the optimal baseline and detector location needs to consider them all.

\section{Acknowledgement}

JE is supported by the Chinese Academy of Sciences Fellowship for Young International Scientists grant number 2010Y2JA01. EC and XZ are supported in part by the NSF of China.

\section{References}

[1] F. P. An et al. [DAYA-BAY Collaboration], "Observation of electron-antineutrino disappearance at Daya Bay," Phys. Rev. Lett. 108 (2012) 171803 [arXiv:1203.1669 [hep-ex]].

[2] D. Dwyer, "Daya Bay Results," presented at Neutrino 2012 in Kyoto. Available at http://neu2012.kek.jp/neu2012/programme.html.

[3] J. K. Ahn et al. [RENO Collaboration], "Observation of Reactor Electron Antineutrino Disappearance in the RENO Experiment," Phys. Rev. Lett. 108 (2012) 191802 [arXiv:1204.0626 [hep-ex]].

[4] G. L. Fogli, E. Lisi, A. Marrone, A. Palazzo and A. M. Rotunno, "Evidence of $\theta_{13 \succsim 0}$ from global neutrino data analysis," Phys. Rev. D 84 (2011) 053007 [arXiv:1106.6028 [hep-ph]]. T. Schwetz, M. Tortola and J. W. F. Valle, "Where we are on $\theta_{13}$ : addendum to 'Global neutrino data and recent reactor fluxes: status of three-flavour oscillation parameters'," New J. Phys. 13 (2011) 109401 [arXiv:1108.1376 [hep-ph]].

[5] Y. Abe et al. [DOUBLE-Chooz Collaboration], "Indication for the disappearance of reactor electron antineutrinos in the Double Chooz experiment," Phys. Rev. Lett. 108 (2012) 131801 [arXiv:1112.6353 [hep-ex]].

[6] M. Apollonio et al. [Chooz Collaboration], "Search for neutrino oscillations on a long baseline at the Chooz nuclear power station," Eur. Phys. J. C 27 (2003) 331 [hepex/0301017]. 
[7] F. Boehm, J. Busenitz, B. Cook, G. Gratta, H. Henrikson, J. Kornis, D. Lawrence and K. B. Lee et al., "Final results from the Palo Verde neutrino oscillation experiment," Phys. Rev. D 64 (2001) 112001 [hep-ex/0107009].

[8] S. T. Petcov and M. Piai, "The LMA MSW solution of the solar neutrino problem, inverted neutrino mass hierarchy and reactor neutrino experiments," Phys. Lett. B 533 (2002) 94 [hep-ph/0112074]. S. Choubey, S. T. Petcov and M. Piai, "Precision neutrino oscillation physics with an intermediate baseline reactor neutrino experiment," Phys. Rev. D 68 (2003) 113006 [hep-ph/0306017].

[9] H. Minakata, H. Nunokawa, S. J. Parke and R. Zukanovich Funchal, "Determination of the neutrino mass hierarchy via the phase of the disappearance oscillation probability with a monochromatic anti-electron-neutrino source," Phys. Rev. D 76 (2007) 053004 [Erratum-ibid. D 76 (2007) 079901] [hep-ph/0701151].

[10] J. Cao, "Observation of $\bar{\nu}_{e}$ Disappearance at Daya Bay," presented at $\nu$ Turn under Gran Sasso. Available at http://agenda.infn.it/contributionListDisplay.py?confId $=4722$.

[11] "Observation of reactor neutrino disappearance at RENO," presented at $\nu$ TURN 2012 under Gran Sasso. Available at http://agenda.infn.it/contributionListDisplay.py?confId $=4722$.

[12] Y. Wang, "Daya Bay II: The Next Generation Reactor Neutrino Experiment" presented at NuFact in Williamsburg, Virginia. Available at https://www.jlab.org/indico/conferenceTimeTable.py?confId=0\#20120725.detailed.

[13] L. Zhan, Y. Wang, J. Cao and L. Wen, "Determination of the Neutrino Mass Hierarchy at an Intermediate Baseline," Phys. Rev. D 78 (2008) 111103 [arXiv:0807.3203 [hep-ex]].

[14] M. Batygov, S. Dye, J. Learned, S. Matsuno, S. Pakvasa and G. Varner, J. Learned, S. T. Dye, S. Pakvasa and R. C. Svoboda, "Determination of neutrino mass hierarchy and $\theta_{13}$ with a remote detector of reactor antineutrinos," Phys. Rev. D 78 (2008) 071302 [hep-ex/0612022]. "Prospects of neutrino oscillation measurements in the detection of reactor antineutrinos with a medium-baseline experiment," arXiv:0810.2580 [hep-ph].

[15] L. Zhan, Y. Wang, J. Cao and L. Wen, "Experimental Requirements to Determine the Neutrino Mass Hierarchy Using Reactor Neutrinos," Phys. Rev. D 79 (2009) 073007 [arXiv:0901.2976 [hep-ex]].

[16] E. Ciuffoli, J. Evslin and X. Zhang, "The Neutrino Mass Hierarchy at Reactor Experiments now that theta13 is Large," arXiv:1208.1991 [hep-ex].

[17] X. Qian, D. A. Dwyer, R. D. McKeown, P. Vogel, W. Wang, and C. Zhang, "Mass Hierarchy Resolution in Reactor Anti-neutrino Experiments: Parameter Degeneracies and Detector Energy Response," arXiv:1208.1551 [hep-ex].

[18] E. Ciuffoli, J. Evslin and X. Zhang, "The High Energy Neutrino Nuisance at a Medium Baseline Reactor Experiment," arXiv:1208.4800 [hep-ph]. 
[19] M. Weng, "Observation of Electron Antineutrino Disappearance at Daya Bay," presented at ICFPC 2012 in Weihai.

[20] M. Apollonio et al. [CHOOZ Collaboration], "Determination of neutrino incoming direction in the $\mathrm{CHOOZ}$ experiment and supernova explosion location by scintillator detectors," Phys. Rev. D 61 (2000) 012001 [hep-ex/9906011].

[21] P. Ghoshal and S. T. Petcov, "Addendum: Neutrino Mass Hierarchy Determination Using Reactor Antineutrinos," arXiv:1208.6473 [hep-ph].

[22] P. Ghoshal and S. T. Petcov, "Neutrino Mass Hierarchy Determination Using Reactor Antineutrinos," JHEP 1103 (2011) 058 [arXiv:1011.1646 [hep-ph]].

[23] P. Vogel and J. F. Beacom, "Angular distribution of neutron inverse beta decay, $\bar{\nu}_{e}+p \rightarrow$ $e^{+}+n, "$ Phys. Rev. D 60 (1999) 053003 [hep-ph/9903554].

[24] H. Nunokawa, S. J. Parke and R. Zukanovich Funchal, "Another possible way to determine the neutrino mass hierarchy," Phys. Rev. D 72 (2005) 013009 [hep-ph/0503283].

[25] P. Vogel and J. Engel, "Neutrino Electromagnetic Form-Factors," Phys. Rev. D 39 (1989) 3378. 